\title{
Traumatismos raquimedulares penetrantes em adolescentes
}

\author{
Penetrating spinal cord injuries in adolescents
}

Traumatismos raquimedulares penetrantes en adolescentes

\author{
Marcelo Campos Moraes Amato' \\ Marcelo Volpon Santos ${ }^{2}$ \\ Alfredo Weltson ${ }^{3}$ \\ Ricardo Santos de Oliveira ${ }^{4}$ \\ José Luiz Romeo Boullosa ${ }^{5}$
}

\section{RESUMO}

Objetivo: analisar a conduta em uma série de adolescentes com traumatismos raquimedulares penetrantes (TRMp) e, confrontando com a literatura, estabelecer a melhor estratégia terapêutica para esses pacientes. Métodos: avaliação retrospectiva de prontuários médicos de dez pacientes, nove do sexo masculino e um do feminino, com TRMp e menores de 18 anos, tratados à nível hospitalar. Analisou-se a anamnese, o exame neurológico e os exames de imagem. A conduta terapêutica variou entre cirurgia ou conservador. Resultados: a média de idade foi de 16 anos (13 a 18 anos). A mediana de hospitalização inicial foi de 11 dias (4 a 180 dias). Quatro pacientes foram submetidos à laminectomia, um ao desbridamento da lesão e os demais a tratamento conservador. Só um paciente, foi submetido à laminectomia, que migrou para Frankel E. Nenhum paciente apresentou infecção da ferida ou instabilidade tardia da coluna vertebral. Conclusão: as lesões medulares se-

\section{ABSTRACT}

Objective: to analyze the management of a series of adolescents with penetrating spinal cord injuries ( $p S C I$ ) and comparing to literature, provide the best therapeutic strategy to these patients. Methods: retrospective evaluation of medical reports of ten consecutive cases of pSCI, nine from the male sex and one from the female. All the patients were under 18 years old. The clinical history, neurologic evaluation and the images exams were analyzed. The therapeutic management varied between surgery and conservative. Results: the average age was 16 years old (form 13 to 18 years). The median time spent at the first hospitalization was 11 days (from 4 to 180 days). Four patients were submitted to laminectomy, one to wound debridement and five to conservative treatment. Only one patient submitted to laminectomy had neurological improvement. No patients presented in this series showed, in the follow-up, wound infection or spinal instability. Conclusion: secondary

\section{RESUMEN}

Objetivo: analizar la conducta en una serie de adolescentes con traumatismos raquimedulares penetrantes (TRMp) y confrontar con la literatura, además de establecer la mejor estrategia terapéutica para eses pacientes. Métodos: evaluación retrospectiva de historias clínicas de diez pacientes, nueve masculinos y un femenino de TRMp menores de 18 años, tratados a nivel hospitalar. Se analizó la anamnesis, el examen neurológico y los exámenes de imagen. La conducta terapéutica varió entre cirugía o tratamiento conservador. Resultados: el promedio de edad fue de 16 años (13 a 18 años). La media de hospitalización inicial fue de 11 dias (4 a 180 días). Cuatro pacientes fueron sometidos al tratamiento conservador. Solo un paciente sometido a laminectomía migró para Frankel E. Ningún paciente presentó infección de la herida o inestabilidad tardía de la columna vertebral. Conclusión: las lesiones medulares secundarias desempeñan poca impor-

\footnotetext{
Trabalho realizado no Hospital das Clínicas da Faculdade de Medicina de Ribeirão Preto da Universidade de São Paulo - USP - São Paulo (SP), Brasil.

'Pós-graduando (Doutorado) em Clínica Cirúrgica da Faculdade de Medicina de Ribeirão Preto da Universidade de São Paulo - USP - São Paulo (SP), Brasil ${ }^{2}$ Médico adido da Disciplina de Neurocirurgia da Hospital das Clínicas da Faculdade de Medicina de Ribeirão Preto da Universidade de São Paulo - USP - São Paulo (SP), Brasil.

${ }^{3}$ Médico residente do Hospital das Clínicas da Faculdade de Medicina de Ribeirão Preto da Universidade de São Paulo - USP - São Paulo (SP), Brasil. ${ }^{4}$ Médico do Serviço de Neurocirurgia Pediátrica do Hospital das Clínicas da Faculdade de Medicina de Ribeirão Preto da Universidade de São Paulo - USP - São Paulo (SP), Brasil.

${ }^{5}$ Médico Assistente do Serviço de Neurocirurgia do Hospital das Clínicas da Faculdade de Medicina de Ribeirão Preto da Universidade de São Paulo - USP - São Paulo (SP), Brasil.
} 
cundárias desempenham pouca importância no resultado neurológico, $\mathrm{o}$ que leva à definição clínica do quadro na admissão hospitalar da maioria dos casos. Houve uma nítida predominância de TRMp em adolescentes do sexo masculino. Não ocorreu diferença significativa entre as condutas realizadas em relação ao resultado neurológico, à infecção e instabilidade. $\mathrm{O}$ tratamento deve ser individualizado, não negligenciando o tratamento clínico e considerando a cirurgia apenas em casos específicos.

\section{DESCRITORES: Traumatismos} da medula espinal/cirurgia; Traumatismos da medula espinal/terapia; Ferimentos perfurantes; Adolescente spinal cord injuries are less relevant to the neurological status, which means that the sequelae are established at hospital admission in most of the cases. There was a clear predominance of pSCI in male adolescents. There was no significant difference between the cases that were managed clinically or surgically, concerning the neurological outcome, infection and instability. The therapy must be individualized, the clinical management must not be neglected and surgery must be considered only in specific cases.

KEYWORDS: Spinal cord injuries/ surgery; Spinal cord injuries/ therapy; Wounds, stab; Adolescent tancia en el resultado neurológico, lo cual lleva a la definición clínica del cuadro en la admisión hospitalar de la mayoría de los casos. Hubo una nítida predominancia de TRMp en adolescentes del sexo masculino. No ocurrió diferencia significativa entre las conductas realizadas en relación al resultado neurológico, infección e inestabilidad. El tratamiento debe ser individualizado, sin negligencia en el tratamiento clínico y considerando la cirugía sólo para casos específicos.

\section{DESCRIPTORES: Traumatismos} de la médula espinal/cirugía; Traumatismos de la médula espinal/terapia; Heridas punzantes; Adolescente

\section{INTRODUÇÃO}

Traumatismos raquimedulares penetrantes (TRMp) são a terceira causa mais comum de lesão da medula espinhal depois de acidentes automobilísticos e quedas ${ }^{1,2}$. Estes ferimentos podem ser causados por arma branca (FAB) ou arma de fogo (FAF) e são entidades nosológicas distintas dos ferimentos não penetrantes. As lesões medulares causadas por ferimentos penetrantes são resultado direto da energia inicial e extensão da penetração, muito mais do que a ação de compressão medular por corpo estranho ou fragmentos ósseos persistentes no canal. Portanto, são consideradas lesões definitivas e não progressivas ${ }^{1,3}$.

A fisiopatologia das lesões ditas secundárias é bem conhecida como a isquemia por transecção de vasos sanguíneos, a falência do mecanismo de autorregulação e os efeitos sistêmicos como a hipotensão ${ }^{1}$, no entanto não está claro qual o grau de lesão secundária existe após um trauma penetrante e quanto de lesão é reversível ou possível de se prevenir com o tratamento na fase aguda ${ }^{3}$.

Portanto, além de conhecer o prognóstico do paciente, o grande desafio desse tipo de lesão é a decisão por uma abordagem conservadora ou cirúrgica ${ }^{1,4-7}$. Há uma preocupação inevitável em melhorar o quadro neurológico do paciente, o qual pode induzir a uma conduta mais agressiva e, às vezes, uma consequente piora clínica. Os fatores que influenciam a decisão de tratamento são o estado neurológico do paciente, o tipo de lesão do canal vertebral $^{4}$ e o material do trauma. Nos FAB, o tamanho da arma branca e a extensão da lesão de outros órgãos contribuem para eventual recuperação neurológica do paciente e para a decisão terapêutica. Entretanto, nos FAF é importante identificar se o projétil foi de baixa ou alta velocidade, sua massa, seu material e os tecidos lesados ${ }^{1,3}$. Para uma análise de comorbidade, deve-se procurar as lesões associadas cranianas, cervicais, pulmonares ou abdominais, e se há algum sinal de instabilidade identificada no alinhamento da coluna ${ }^{4}$.

O objetivo deste trabalho é analisar a conduta em uma série de adolescentes com TRMp e, confrontando com a literatura, estabelecer a melhor estratégia terapêutica para esses pacientes.

\section{MÉTODOS}

Análise retrospectiva do seguimento de dez casos consecutivos de TRMp em pacientes menores de 18 anos, atendidos no Hospital das Clínicas de Ribeirão Preto da Universidade de São Paulo, de 1989 a 2008.

Analisou-se a anamnese e o exame neurológico dos pacientes, classificando-os segundo o mecanismo de trauma, a escala de Frankel e a Glasgow Outcome Scale (GOS) para avaliar a capacidade funcional durante o seguimento: 1 , morto; 2 , estado vegetativo; 3 , incapacidade grave; 4, incapacidade moderada; e 5, boa recuperação. Os pacientes foram submetidos a radiografias (RX) estáticas e tomografias computadorizadas (TC) para avaliar a posição do projétil, a presença de fragmentos ósseos, a trajetória do evento e os sinais de instabilidade da coluna. Radiografias dinâmicas em flexão e extensão foram realizadas na ausência de dor, após a realização da TC para avaliar a estabilidade da coluna. A definição utilizada para coluna estável é esta poder suportar peso fisiológico sem que ocorra deformidade estrutural, dor e/ou déficits neurológicos ${ }^{3}$.

A conduta terapêutica variou conforme o médico responsável pelo atendimento. Os critérios para indicar 
tratamento cirúrgico foram a presença de fragmentos ósseos ou de projéteis no canal vertebral e a suspeita de laceração dural pela imagem ou mecanismo de trauma.

\section{RESULTADOS}

Os resultados estão apresentados na Tabela 1 e as figuras mostram casos representativos (Figuras 1 a 3 ).

Dos dez pacientes apenas um era do sexo feminino. Esta sofreu FAB e o restante FAF. A média de idade foi de 16 anos no momento do trauma. Seis pacientes se apresentaram com Frankel A, um com B, dois com D e um com E. Com exceção da paciente feminina que não teve alta por motivos sociais, a mediana de hospitalização inicial foi de 11 dias.

Não houve relato de fístula liquórica de alto débito nos pacientes. Os quatro pacientes que foram submetidos à laminectomia tiveram como objetivo principal a retirada de fragmentos ósseos e corpo estranho dentro do canal. No entanto, quando observada laceração dural, esta foi reparada. A paciente que sofreu FAB foi submetida apenas ao desbridamento da ferida. Os demais pacientes foram submetidos a cuidados locais com o ferimento e tratamento clínico. Apenas um paciente apresentou melhora neurológica. Ele chegou em Frankel D, foi submetido à laminectomia e progrediu para Frankel E.

Quatro pacientes tiveram traumas associados, dois torácicos e dois múltiplos. Um destes apresentou comprometimento torácico grave que levou a seis meses de tempo de internação e óbito por choque séptico, quatro meses após a alta. Nenhum paciente apresentou infecção da ferida traumática ou operatória.

Nenhum paciente desta série apresentou instabilidade da coluna vertebral e consequente necessidade de fixação ao longo do seguimento estudado.

\section{DISCUSSÃO}

Até os dez anos de idade existem diferenças anatômicas da coluna infantil que influem na incidência e nos tipos de TRM, porém em adolescentes a epidemiologia e o comportamento dos TRM são semelhantes aos dos adultos ${ }^{3,8}$. Observou-se nesta série que ocorreu uma predominância do sexo masculino semelhante à literatura revisada 9 .

A capacidade funcional e a morbimortalidade após o evento traumático, medidos pela escala de Frankel, relacionam-se com a presença de traumatismos associados. Muitos autores concluem que o nível de lesão inicial e a gravidade dos déficits determinam o resultado neurológico muito mais do que o método de tratamento. Também mostram que a descompressão imediata do canal medular, a retirada de fragmentos ósseos ou de projétil e o fechamento de lacerações da dura apresentam resultados desapontadores, o que equivale a afirmar que o resultado neurológico não é afetado por intervenções cirúrgicas precoces ${ }^{3-6,10-12}$. De fato, no presente estudo todos os pacientes com lesão Frankel A ou B não apresentaram melhora neurológica apesar do tratamento instituído. Apenas um paciente com
Frankel D apresentou melhora da força durante o seguimento. Esses estudos desestimularam a intervenção precoce em pacientes com ferimentos penetrantes na coluna em vários serviços. Na literatura observa-se que menos de $4 \%$ dos pacientes são submetidos à exploração cirúrgica ${ }^{13}$. Devido a esse conhecimento, ocorre a ênfase do tratamento na utilização precoce de órteses, hidratação, manejo da infecção, cuidados pulmonares e fisioterapia ${ }^{3}$.

A descompressão cirúrgica e o desbridamento dos ferimentos penetrantes da coluna não parecem alterar o controle da infecção. Em séries maiores, a remoção do projétil e o desbridamento da coluna não diminuíram a incidência de infecção $0^{5,10-12,14,15}$. Dos pacientes deste estudo nenhum evoluiu com infecção no local do ferimento, independente do tipo de tratamento instituído. Aryan et al. mostraram que a intervenção cirúrgica da coluna associada à correção cirúrgica de lesões viscerais está relacionada com maior índice de complicações, do que a reparação isolada da víscera e antibióticos de amplo espectro somente sem manipulação da coluna ${ }^{3}$.

A instabilidade da coluna, conforme mostra a literatura $^{1,3}$, é incomum em casos de ferimentos penetrante, o que torna rara a necessidade de cirurgias para fixação. Simpson, Venger e Narayan reportaram instabilidade em apenas 3 de 142 pacientes com FPC por arma de fogo ${ }^{7}$. No entanto, a laminectomia realizada em pacientes com lesão da coluna anterior ou média por TRMp pode levar à instabilidade adicional e progressiva, ou escoliose, as quais necessitem de fixação cirúrgica durante o seguimento ${ }^{1,11}$. Portanto, deformidade estrutural pode ocorrer de modo paradoxal com o manejo cirúrgico dos TRMp da coluna. Neste estudo, quatro pacientes foram submetidos à laminectomia, mas nenhum apresentou instabilidade da coluna durante o seguimento clínico. A técnica operatória descrita por Jacobs e Berg, na qual a laminectomia deve ser realizada no nível acometido e com boa visualização de um nível acima e outro abaixo ${ }^{6}$, foi descrita em um contexto de ferimentos de guerra e não se aplica aos TRMp que ocorrem em civis $^{3}$.

Há consenso de que fístulas persistentes, mais frequentes em FAB, necessitam de reparação ${ }^{8}$, assim como déficits neurológicos progressivos secundários ao efeito compressivo de projétil retido; déficits secundários a fragmento ósseo ou herniação discal devem ser tratados do mesmo modo que em ferimentos não penetrantes ${ }^{1}$. Hematoma epidural agudo ou abscesso epidural são indicações de cirurgia de urgência ${ }^{1,6,10,11}$. As complicações tardias de TRMp, assim como seringohidromielia (secundária à aracnoidite), migração do projétil dentro do saco tecal e dor intratável podem ser beneficiadas com o tratamento cirúrgico ${ }^{1,10}$.

Nos casos de FAB, Shahlaie, Chang e Anderson sugerem exploração cirúrgica na fase aguda para remoção de material e irrigação da ferida com a justificativa de haver melhora dos déficits neurológicos e das consequências infecciosas ${ }^{16}$. A presença de apenas um traumatismo por arma branca no presente estudo é consistente com os dados da literatura, os 


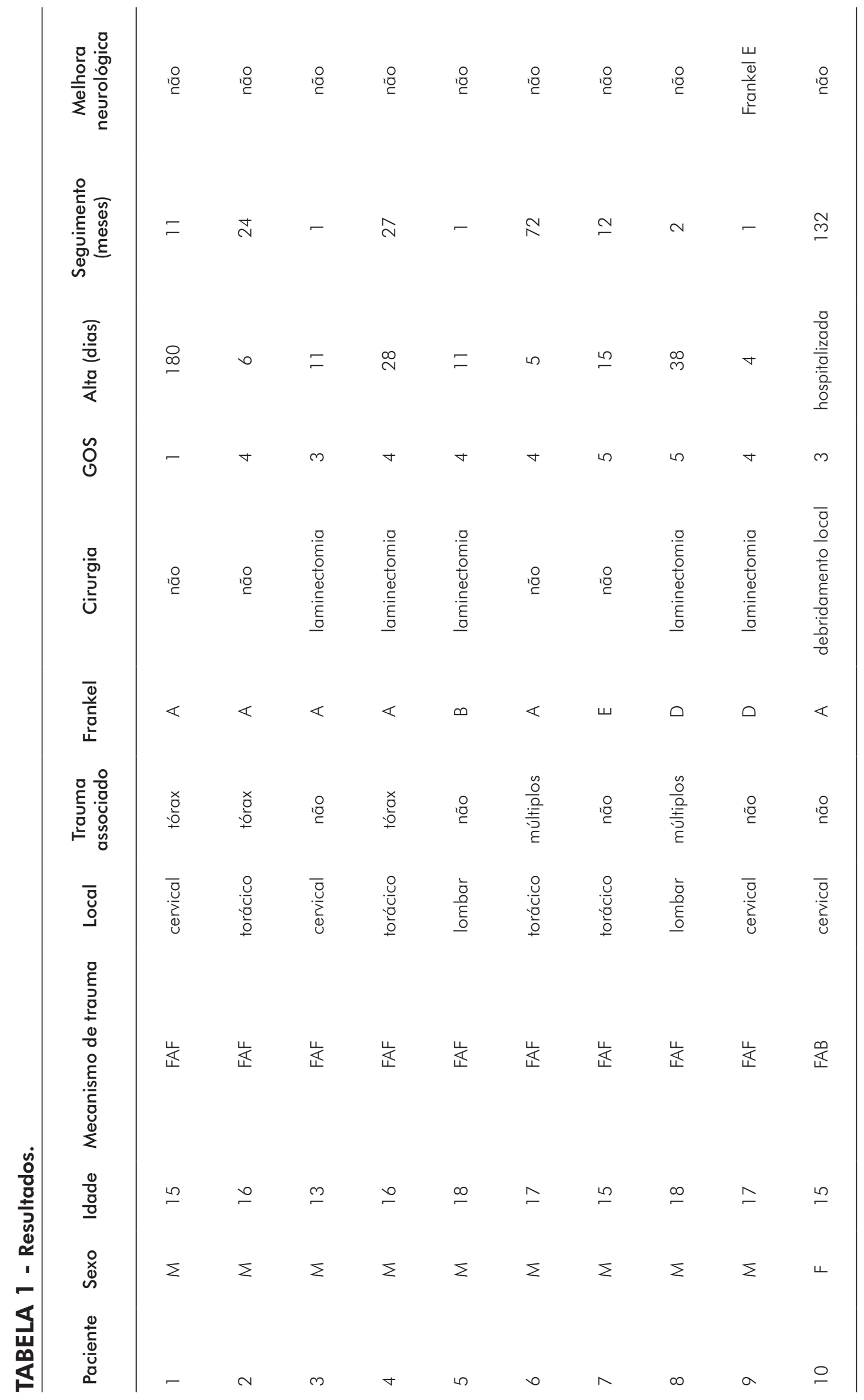



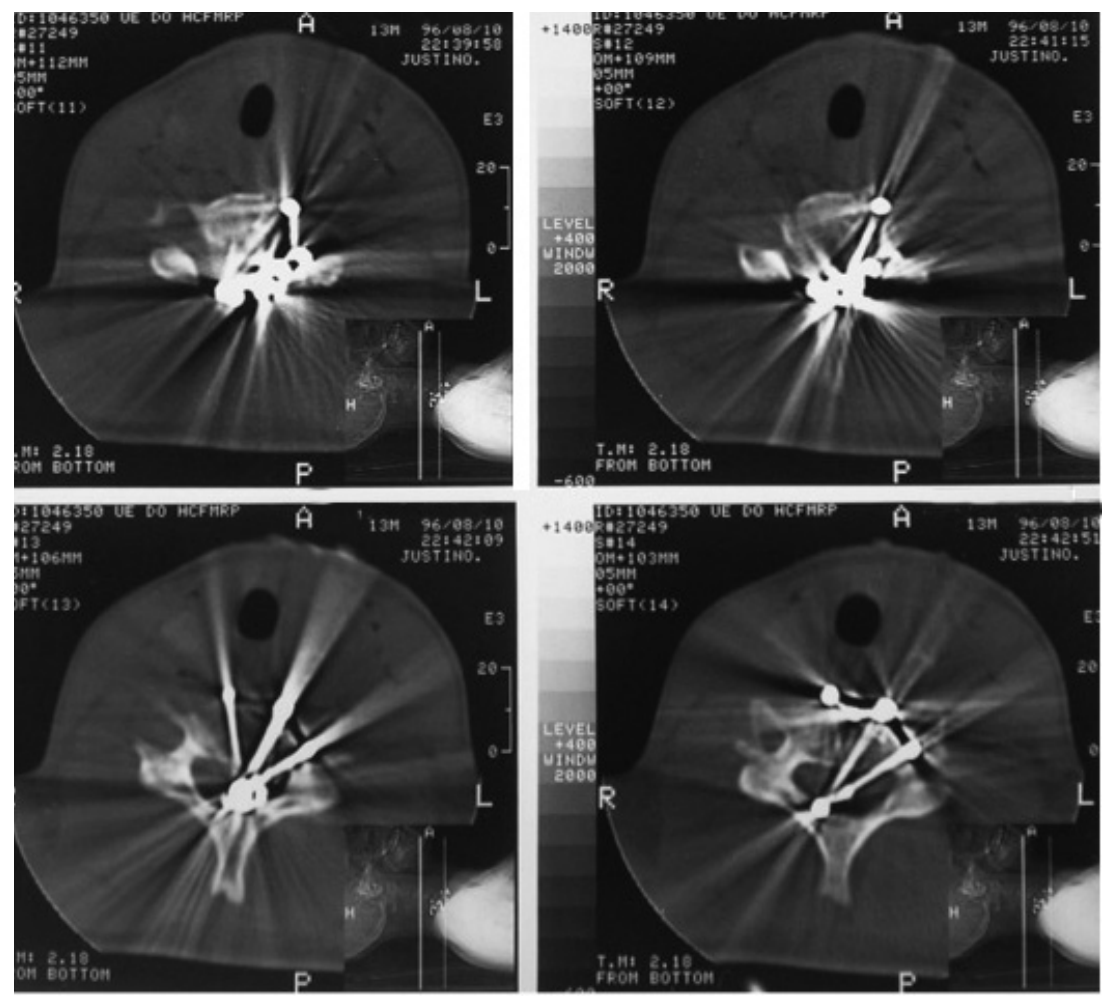

Figura 1

Cortes axiais de TC da janela óssea de paciente (4) que sofreu FAF cenvical. As imagens mostram fragmentos do projétil dentro do canal. O paciente chegou em Frankel A, foi submetido à laminectomia cervical sem fixação, não apresentou melhora neurológica, mantendo-se tetraplégico e com GOS de 3. Não apresentou infecção pós-operatória e nem instabilidade após a laminectomia.

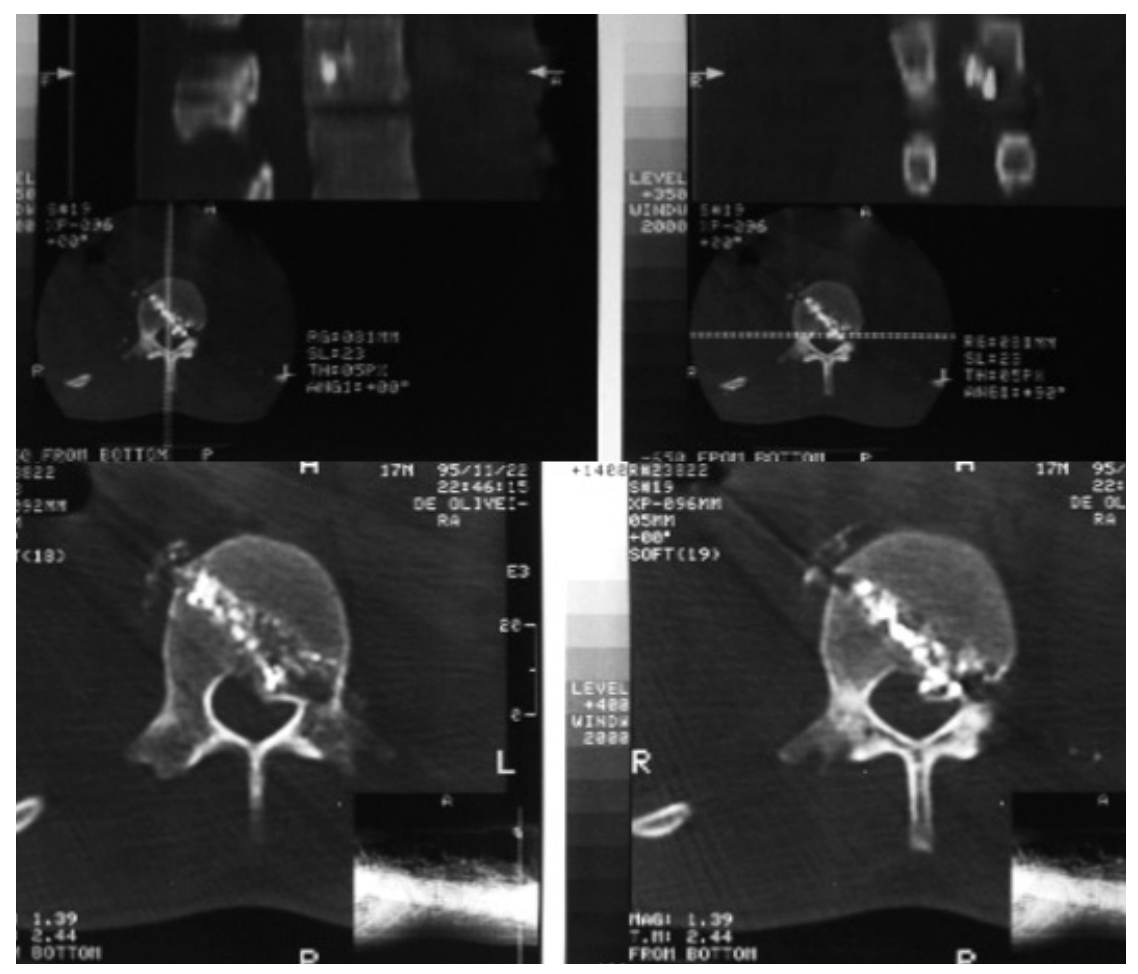

Figura 2

Cortes axiais de TC da janela óssea de paciente (7) que foi vítima de FAF torácico mostra transfixação do corpo vertebral pelo projétil. Este paciente chegou em Frankel A e com abdome agudo, foi submetido à laparotomia sem intervenção na coluna. Manteve a paraplegia durante o seguimento e GOS de 4. Não apresentou infecção e nem instabilidade da coluna. 


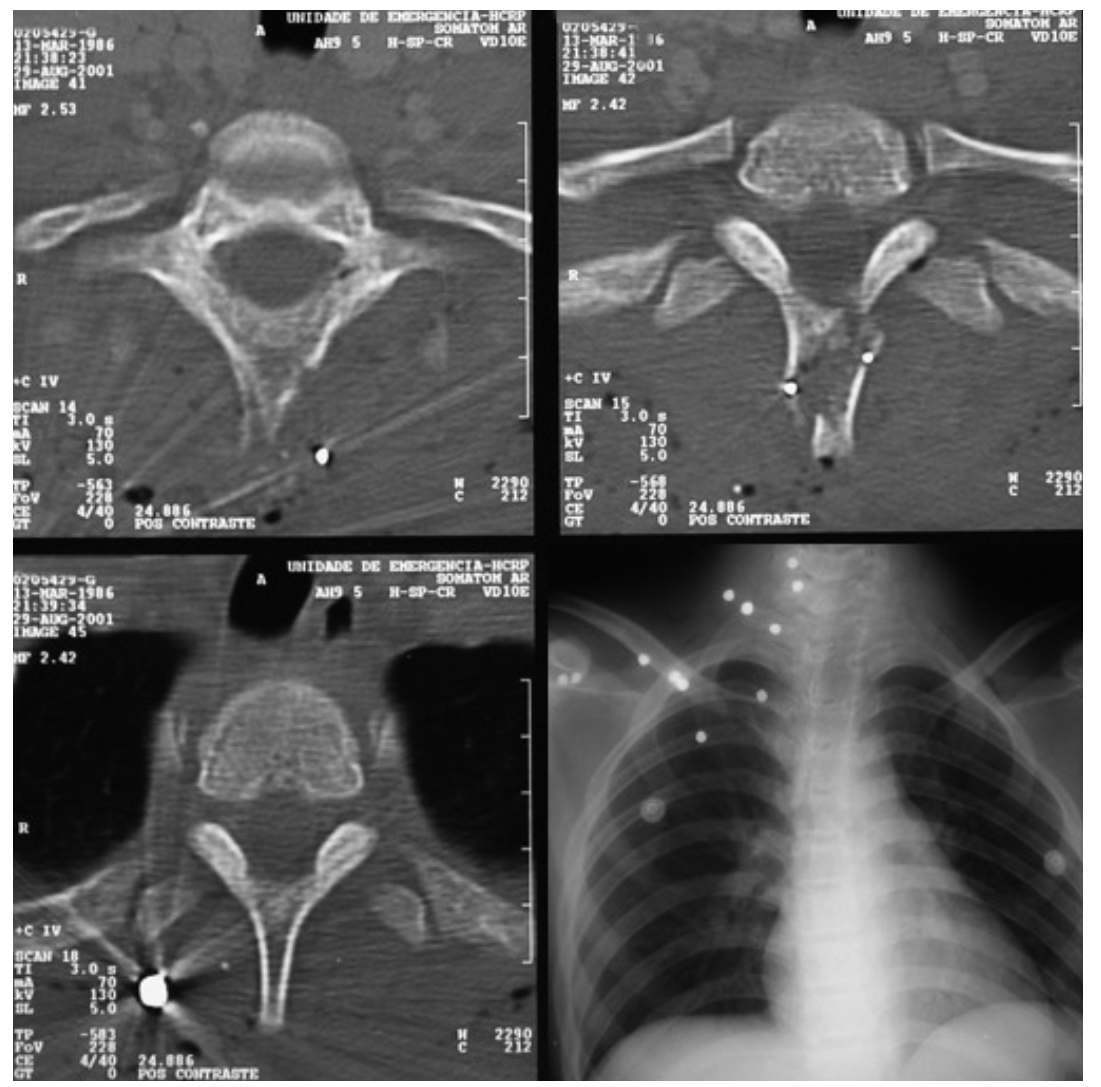

Figura 3

Cortes axiais de TC da janela óssea mostrando projétil paravertebral e fratura do processo espinhoso da primeira vértebra torácica. O raio X mostra múltiplos projéteis alojados no subcutâneo. Este paciente (8) foi submetido a tratamento conservador. Chegou em Frankel E, manteve-se com exame neurológico normal durante o seguimento e GOS de 5.

quais apontam menor frequência deste tipo entre os TRMp. Mais raro ainda são os casos que apresentam fragmentos ou a própria arma branca retida ${ }^{16}$, e não nos permite concluir qual o melhor tratamento para esses casos específicos.

Aryan et al. apresentam uma série de 60 adolescentes e defendem a abordagem conservadora, tendo em vista os argumentos supracitados ${ }^{3}$. No presente estudo, cinco pacientes foram submetidos a abordagens cirúrgicas e não apresentaram pior controle de infecção ou instabilidade da coluna. Em vigência dos dados apresentados, o tratamento deve ser individualizado e a abordagem cirúrgica pode ser indicada, com o preceito básico de primum non nocere.

O tratamento clínico também é limitado. Os pacientes devem ser mantidos com antibióticos de amplo espectro e boa penetração da barreira hematoencefálica, como cefalosporinas de terceira geração de 10 a 14 dias $^{1,10,11,14}$. A profilaxia do tétano deve ser administrada aos pacientes que não estão em dia com a vacinação. Heparina de baixo peso molecular para evitar trombose venosa profunda é padrão no manejo de FPC. Manutenção da pressão arterial sistêmica em níveis normais para garantir a perfusão medular é crucial para minimizar os danos secundários à medula. $\mathrm{O}$ choque circulatório deve ser manejado prontamente com hidratação e agentes inotrópicos, de forma que garanta suprimento sanguíneo suficiente para a medula espinhal ${ }^{11}$. Visto as controvérsias recentes com relação ao uso de corticosteroides em traumatismo raquimedular e a falta de evidência em TRMp, estes não devem ser utilizados. Seu uso leva a maior prevalência de infecções da ferida ${ }^{17}$, risco teórico aumentado de hemorragia intestinal ${ }^{18,19} \mathrm{e}$ risco aumentado de hiperglicemia, necrose de quadril. Altas doses de corticosteroides podem também impedir a recuperação e a regeneração neurológica ${ }^{18,20}$.

\section{CONCLUSÃO}

Os TRMp na população pediátrica são graves com altos índices de sequela neurológica definitiva e as lesões medulares secundárias desempenham pouca importância no resultado neurológico, o que leva à definição do quadro logo à admissão na maioria dos casos.

No presente estudo ocorreu uma nítida predominância de TRMp em adolescentes do sexo masculino. Não houve diferença significativa entre os casos operados e não operados com relação ao resultado neurológico, à infecção e instabilidade. Portanto, deve-se individualizar cada caso e estudar o eventual benefício e efeitos adversos de intervenção cirúrgica. Os cuidados clínicos, apesar de pouco influenciarem no resultado final, não podem ser negligenciados, evitando assim eventuais danos secundários e piora neurológica. 


\section{REFERÊNCIAS}

1. Jallo GI. Neurosurgical management of penetrating spinal injury. Surg Neurol. 1997;47(4):328-30.

2. Kalsbeek WD, McLaurin RL, Harris BS 3rd, Miller JD. The National Head and Spinal Cord Injury Survey: major findings. J Neurosurg. 1980;Suppl:S19-31.

3. Aryan HE, Amar AP, Ozgur BM, Levy ML. Gunshot wounds to the spine in adolescents. Neurosurgery. 2005;57(4):748-52.

4. Duz B, Cansever T, Secer HI, Kahraman S, Daneyemez MK, Gonul E. Evaluation of spinal missile injuries with respect to bullet trajectory, surgical indications and timing of surgical intervention: a new guideline. Spine (Phila Pa 1976). 2008;33(20):E746-53.

5. Aarabi B, Alibaii E, Taghipur M, Kamgarpur A. Comparative study of functional recovery for surgically explored and conservatively managed spinal cord missile injuries. Neurosurgery. 1996;39(6):1133-40.

6. Jacobs GB, Berg RA. The treatment of acute spinal cord injuries in a war zone. J Neurosurg. 1971;34(2 Pt 1):164-7.

7. Simpson RK Jr, Venger BH, Narayan RK. Treatment of acute penetrating injuries of the spine: a retrospective analysis. J Trauma. 1989;29(1):42-6.

8. Boullosa JLR. Traumatismo raquimedular na infância e na adolescência. In: Oliveira RS, Machado HR. Neurocirurgia Pediátrica. Fundamentos e Estratégias. Rio de Janeiro: DiLivros; 2009. p. 155-68.
9. Stover SL, Fine PR. The epidemiology and economics of spinal cord injury. Paraplegia. 1987;25(3):225-8.

10.Heiden JS, Weiss MH, Rosenberg AW, Kurze T, Apuzzo ML. Penetrating gunshot wounds of the cervical spine in civilians. Review of 38 cases. J Neurosurg. 1975;42(5):575-9.

11.Stauffer ES, Wood RW, Kelly EG. Gunshot wounds of the spine: the effects of laminectomy. J Bone Joint Surg Am. 1979;61(3):389-92.

12. Yashon D, Jane JA, White RJ. Prognosis and management of spinal cord and cauda equina bullet injuries in sixty-five civilians. J Neurosurg. 1970;32(2):163-70.

13.Rea GL. Subaxial injuries of the cervical spine. In: Menezes AH, Sonntag VKH. Principles of Spinal Surgery. New York, McGrawHill, 1996. p. 885-98.

14.Roffi RP, Waters RL, Adkins RH. Gunshot wounds to the spine associated with a perforated viscus. Spine (Phila Pa 1976). 1989;14(8):808-11.

15.Romanick PC, Smith TK, Kopaniky DR, Oldfield D. Infection about the spine associated with low-velocitymissile injury to the abdomen. J Bone Joint Surg Am. 1985;67(8): 1195-201.

16.Shahlaie K, Chang DJ, Anderson JT. Nonmissile penetrating spinal injury. Case report and review of the literature. J Neurosurg Spine. 2006;4(5):400-8.
17.Bracken MB, Collins WF, Freeman DF, Shepard MJ, Wagner FW, Silten RM, et al. Efficacy of methylprednisolone in acute spinal cord injury. JAMA. 1984;251(1): 45-52.

18.Bracken MB, Shepard MJ, Collins WF Jr, Holford TR, Baskin DS, Eisenberg $\mathrm{HM}$, et al. Methylprednisolone or naloxone treatment after acute spinal cord injury: 1-year follow-up data. Results of the second National Acute Spinal Cord Injury Study. J Neurosurg. 1992;76(1):23-31.

19.Levy ML, Gans W, Wijesinghe HS, SooHoo WE, Adkins RH, Stillerman CB. Use of methylprednisolone as an adjunct in the management of patients with penetrating spinal cord injury: outcome analysis. Neurosurgery. 1996;39(6):1141-8; discussion 1148-9.

20.Bracken MB, Holford TR. Effects of timing of methylprednisolone or naloxone administration on recovery of segmental and long-tract neurological function in NASCIS 2. J Neurosurg. 1993;79(4):500-7.

\section{Correspondência}

Marcelo Campos Moraes Amato

Rua Bernardino de Campos, 435, apto. 402

CEP: 14015-130 - Ribeirão Preto (SP), Brasil.

Tel: (16) 8148-6886/Fax: (16) 3632-8234

E-mail:marcelo@amato.com.br 